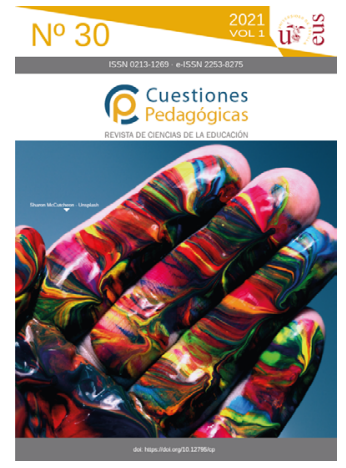

\title{
Recursos culturales y etnoeducación: propuesta para el desarrollo de cultura de paz
}

Cultural resources and ethno-education: a proposal for the development of a culture of peace

Recibido: 13/11/2020 | Revisado: 05/05/2021 | Aceptado: 08/06/2021 | Online first: 10/06/2021 | Publicado: 01/07/2021

\section{Olga Lucía Arbeláez-Rojas}

Universidad Pontificia Bolivariana (Colombia)

olga.arbelaez@upb.edu.co

https://orcid.org/0000-0003-2732-4999

Adriana del Socorro Álvarez-Correa

Universidad Pontificia Bolivariana (Colombia)

adriana.alvarez@upb.edu.co

https://orcid.org/0000-0003-0212-0025

\section{Alejandro Londoño-Hernández}

Universidad Pontificia Bolivariana (Colombia)

alejandro.londonoh@upb.edu.co

https://orcid.org/0000-0002-7507-4683
Resumen: Tras el acuerdo de paz firmado entre el gobierno de Colombia y las FARC-EP en el año 2016, el país ha entrado en un periodo histórico que requiere de diversos análisis y reflexiones sobre la violencia que ha padecido y las posibilidades de construir la paz. Tarea mayor para la educación en una sociedad que, a pesar de las desigualdades, divisiones y polarización, es rica en experiencias de mediación y transformación del conflicto gracias a su diversidad social, étnica y cultural. Este ensayo, derivado de la investigación Creando paz: recursos culturales en experiencias de mediación y gestión constructiva de conflictos y su aporte a la formación de competencias ciudadanas y construcción de cultura de paz, sostiene cómo la formación en la Licenciatura en Etnoeducación de la Universidad Pontificia Bolivariana, en convenio con el Instituto Misionero de Antropología, constituye una alternativa para el desarrollo de una cultura de paz a través del reconocimiento de recursos culturales y la formación en estos. El proyecto educativo se basa en la idea de que educarse en el respeto, el diálogo y el reconocimiento transforma la visión de mundo, desarrolla capacidades, incorpora valores que redundan en conductas que favorecen la manifestación de recursos culturales, es decir, del repertorio de elementos que hacen parte de la identidad y la memoria colectiva de los pueblos. Se hace referencia a
Abstract: After the peace agreement settled by the National Government and FARC, the Colombian nation has entered an epoch that requires diverse analysis and reflection over the violence suffered by people and the forthcoming possibilities for building peace. A fundamental task for Education within a society that, despite inequality, division, and polarization, has been rich in mediation and conflict transformation experiences. Derived from the research 'Creando paz: cultural resources in mediation and constructive management of conflict, and its contribution to Civil Skills training and the construction of a culture of peace', this essay sets out the curriculum of Ethno-Education Partnership Program held by Universidad Pontificia Bolivariana and Instituto Misionero de Antropología as a proposal to achieve a peace culture based on the recognition of cultural resources and education about what they are and mean. This educational project is based on the idea that educating oneself in respect, dialogue and recognition transforms the vision of the world, develops capacities, incorporates values that result in behaviors that favor the manifestation of cultural resources, that is, of the repertoire of elements that are part of the identity and collective memory of the peoples. Social and education issues in Colombia are referred, Ethno-education is analyzed as a public policy, to finally focus on this program educational 
problemáticas de índole social y educativo en Colombia, se analiza cómo la etnoeducación se ha constituido como política pública, y se pone el foco en la experiencia formativa de la licenciatura en relación con la categoría recursos culturales como propuesta para el desarrollo de cultura de paz.

Palabras clave: educación intercultural, etnoeducación, plan de estudios, recursos culturales, cultura de paz. experience in relation to cultural resources category as a proposal for building peace.

\section{Introducción}

En el año 2015, el Departamento Administrativo de Ciencia, Tecnología e Innovación de Colombia (Colciencias) lanzó una convocatoria con el propósito de "conformar un banco de proyectos de investigación que aporten a la equidad, la paz y la educación desde el conocimiento que generen y transformen, ámbitos que se identifican como prioritarios en el actual Plan Nacional de Desarrollo (PND)" (Colciencias, 2015, p. 1). La Universidad Pontificia Bolivariana, en conjunto con el grupo de investigación Ciudadanías, Conflictos y Políticas Sociales de la Corporación Región, respondió a dicha convocatoria con el proyecto Creando paz: recursos culturales en experiencias de mediación y gestión constructiva de conflictos y su aporte a la formación de competencias ciudadanas y construcción de cultura de paz.

El objetivo general de la investigación -analizar el uso de recursos culturales en casos de mediación y otras formas de gestión constructiva de conflictos, sus aportes a la formación de competencias ciudadanas y la construcción de culturas de paz- así como sus objetivos específicos, apuntan a desafíos estratégicos planteados por el Ministerio de Educación Nacional $(2007,2017)$ en los planes nacionales decenales de educación (PNDE), tales como:

, El establecimiento de lineamientos curriculares generales, pertinentes y flexibles;

, La construcción de una política pública para la formación de educadores;

> Construir una sociedad en paz sobre una base de equidad, inclusión, respeto a la ética y equidad de género (PNDE 2016-2026) (Ministerio de Educación Nacional, 2017, p. 17), y construir una "educación en y para la paz, la convivencia y la ciudadanía" (PNDE 2006-2016) (Ministerio de Educación Nacional, 2007, p.7).

El problema de la investigación plantea que la violencia, la inadecuada gestión de conflictos y la guerra se concretan en desarticulaciones, entre las cuales se destaca la falta de coherencia entre el sistema educativo nacional y el currículo. Desarticulación que ya había sido advertida por el informe Colombia, al filo de la oportunidad elaborado por la Misión de Ciencia, Educación y Desarrollo (Presidencia de la República y Colciencias, 1996). Es en este marco en el cual se propone la revisión del currículo de la Licenciatura en Etnoeducación -que ofrece la Universidad Pontificia Bolivariana en convenio con el Instituto Misionero de Antropología- como una propuesta para el desarrollo de cultura de paz, al concretarse en la identificación, aprendizaje, valoración, intercambio y formación de recursos culturales.

\section{Desarticulación social}

Colombia es un país cuyas prácticas políticas, religiosas y culturales han sido tradicionalmente jerarquizadas y refractarias al cambio, donde se ha conformado una sociedad con una marcada diferenciación social. A causa de esto, ser un país multicultural y diverso, constituido por 65 etnias indígenas, pueblos rom, afrocolombianos y mestizos, la colombiana es una sociedad fragmentada, atravesada por fronteras simbólicas de etnia, estrato y ubicación (rural o urbana), así como por diferencias en factores socioeconómicos y educativos, entre otros. Esta fragmentación se traduce en desigualdades que afectan la competitividad y la movilidad social (Kauffman, 2015; Albán y Rendón, 
2018; González, 2019; Ávila, 2019), así como la percepción y la construcción de los acontecimientos y de la realidad en general.

A ello se suma que Colombia ha padecido el conflicto armado más prolongado en el tiempo y con mayor afectación a la población civil, como advierte el Centro Nacional de Memoria Histórica (Grupo de Memoria Histórica, 2013). Quizá por ello el conflicto tiende a experimentarse con prevalencia de su acepción negativa (como combate, lucha, pelea, enfrentamiento armado), contrario a asumirlo desde una doble dimensión -política y social- como expresión de distintas formas de existencia y relacionamiento, lo que implica, por una parte, la necesidad de incorporar, desde una perspectiva intercultural, la diversidad en la identidad nacional, y por otra, trabajar en el desarrollo de una educación para una ciudadanía capaz de responder a las demandas de una democracia participante, que fomente la convivencia en paz y el desarrollo con enfoque diferencial territorial.

Precisamente, la diversidad étnica, cultural, lingüística y en los niveles de desarrollo de los asentamientos rurales y urbanos, entre otros factores, conllevan a que el conflicto sea una experiencia que se narra de distintas maneras. Así, mientras para unos colombianos se trata de un conflicto que involucra solo a ciertos sectores oficiales y no oficiales, para otros, se trata de una guerra que ha cambiado sus vidas y cuya afectación es permanente e inacabada. Como lo documenta el Centro Nacional de Memoria Histórica, son los habitantes de las zonas rurales quienes se han visto más expuestos a los efectos de una guerra en pocas ocasiones declarada como tal:

Todos los informes ilustran la gradual convergencia entre la guerra y el problema agrario (despojos violentos, concentración ociosa de la tierra y usos inadecuados, colonizaciones y titulaciones fallidas). Pero a los viejos problemas se suman otros nuevos, que muestran las dinámicas inauguradas por el narcotráfico, la explotación minera y energética, los modelos agroindustriales y las alianzas criminales entre paramilitares, políticos, servidores públicos, élites locales económicas y empresariales y narcotraficantes (Grupo de Memoria Histórica, 2013, p. 21).

\section{Desarticulación educativa}

Dos de las leyes vigentes y estructurales del sistema de educación en Colombia, Ley 115 de 1994 -"por la cual se expide la ley general de educación" - y la Ley 30 de 1992 -por la cual se organiza el servicio público de la Educación Superior"-, coinciden en la declaración de algunos términos como autonomía, libertad de pensamiento, pluralismo ideológico, pluralidad, formación integral, derecho, paz y democracia, entre otros. Como puede constatarse, tanto la Ley 30 como la Ley 115 en el artículo primero hacen referencia a la educación como proceso permanente que posibilita el desarrollo integral de las personas. De igual forma, en los artículos $4^{\circ}$ y $1^{\circ}$, respectivamente, presentan a la educación como un servicio público. En los artículos $4^{\circ}$ (Ley 30 ) y $5^{\circ}$ (Ley 115) se menciona la formación en el pensamiento pluralista teniendo en consideración la diversidad étnica como fundamento de la nacionalidad del país. Y, en los artículos $6^{\circ}$ (Ley 30) y $5^{\circ}$ (Ley 115) se invoca a la formación para la participación en la toma de decisiones en las dimensiones económica, política, administrativa y cultural de la nación.

Sin embargo, la crítica histórica al sistema educativo colombiano ha sido que éste es todo lo contrario de un sistema: especie de escalera que transita con obstáculos de lo inferior a lo superior; ascender un escalón no se percibe como resultado del apoyo indeclinable del anterior, sino que es el paso solitario que permite juzgar la carencia del antepuesto y la necesidad de olvidarlo para continuar ascendiendo: la educación media juzga la básica, la técnica y la tecnológica desdicen de la media, la universitaria deslegitima la anterior, y, en general, se hace una fuerte separación entre lo teórico y lo práctico (Luque, Gómez, Reyes, Ramírez Carvajal y Londoño, 1999).

Llama la atención que, precisamente, en dos intentos de reforma que ha tenido la Ley 30 en 2011 y en 2018- (Linares, 2011; Ospina y Suárez, s.f.) la discusión haya estado centrada en los factores de autonomía, financiación y en la carencia de sistematicidad entre los niveles del sistema educativo colombiano. Al respecto es preciso observar que la tentativa gubernamental más sostenida 
en el tiempo para articular el sistema ha sido la aplicación de las pruebas estandarizadas llamadas Pruebas Saber. Por esta razón, a partir del año $2015^{1}$, se empezó a valorar el nivel de aporte relativo de la formación en un subsistema y el paso por el siguiente. Pero esta tentativa, que pretendía responder al compromiso del sistema con los estándares internacionales, no parece mostrar resultados en su compromiso social y con los distintos grupos culturales que constituyen al país.

Ya a mediados de la última década del siglo anterior, la Misión de Ciencia, Educación y Desarrollo (Presidencia de la República y Colciencias, 1996) describía el estado del sistema educativo señalando características, algunas de las cuales permanecen: altas tasas de repitencia, deserción, deficiencia docente, deficiencia pedagógica, infraestructura y materiales inadecuados, indisciplina y falta de educación para la democracia y la competencia, inexistencia de un currículo integrador, falta de información actualizada y materiales. Además de ser un sistema que promueve la enseñanza fragmentada, acrítica, desactualizada e inadecuada.

En síntesis, según la Misión, esta ineficiencia de la educación formal en los niveles de básica primaria y secundaria tiene impactos negativos en la educación superior y en aspectos como la eficiencia y efectividad de los sectores productivo, científico y tecnológico; elementos civilizadores, desempeño cultural y cívico de la población; calidad de la fuerza laboral; falta de valores de solidaridad, convivencia pacífica, respeto por la vida y la equidad. Contrario a esto, de acuerdo con los comisionados, quienes redefinieron el concepto de desarrollo humano en términos de un nuevo ethos cultural ${ }^{2}$, la meta del sistema educativo habría de ser el óptimo desarrollo del saber, la dignidad humana, la solidaridad colectiva y la conciencia social y ecológica:

el avance de la ciencia, la tecnología y la educación supone un nuevo ethos cultural, que se oriente a superar pobreza, violencia, injusticia, intolerancia y discriminación, problemas en donde se encuentra la raíz del atraso socio-económico, político y cultural de Colombia (Presidencia de la República y Colciencias, 1996, p. 31).

De acuerdo con el texto de la Misión, alcanzar dicha meta solo es posible "si se transforman las estructuras fundamentales y se suministran elementos que permitan la mejor y más pertinente aplicación del saber, elementos que puedan adaptarse a situaciones reales en continua transformación" (Presidencia de la República y Colciencias, 1996, p. 36). Todo lo cual exigía consenso sobre cuáles habrían de ser los estándares educativos de los sistemas occidentales avanzados que harían parte del sistema educativo colombiano, pero también cómo se incorporarían los estándares de los sistemas regionales y de los autóctonos e indígenas.

En este último aspecto subyace la ausencia de convergencia, las brechas a las que se hace alusión en el proyecto Creando paz ${ }^{3}$ (Universidad Pontificia Bolivariana y Corporación Región, 2016), y es que no se puede hablar del sistema educativo sin entender el papel del currículo pues el sistema y el currículo se codeterminan.

\section{El currículum y la Etnoeducación en Colombia}

El concepto de currículo se entiende acá en el marco de políticas internacionales que promovieron la modernización de los sistemas educativos, especialmente para los países de América Latina y el Caribe en la década de 1990. Se asume de esta manera en función de un marco de comprensión que permita entender la relación entre el sistema educativo colombiano y las desarticulaciones a las que se busca responder con la investigación Creando paz.

1 Cfr. Guía para la interpretación del reporte de resultados en Saber Pro: medidas de aporte relativo y otros indicadores de calidad de las instituciones de educación superior (Icfes, 2015). https://bit.ly/36wRKzF

2 "El proyecto civilizador debe dar un nuevo sentido y significado a la vida colectiva de los colombianos, con culturas de paz y convivencia, y esto debe gestarse con la participación equitativa de toda la nación" (Presidencia de la República y Colciencias, 1996, p. 43).

3 http://www.creandopaz.org/ 
Como advierte Martínez Boom (1995), desde los años 1950 las formas de pensamiento educativo desbordan las fronteras nacionales al inscribirse en los procesos de mundialización; afirma que "no siempre se ha enseñado con currículo" (p. 173). Como concepto y como práctica, el currículo aparecería ligado a los discursos del desarrollo y la planificación. De manera que el currículo en las reformas educativas procede de la organización científica del trabajo para lograr eficiencia, eficacia, rentabilidad y control social.

El autor compara el papel del plan de estudios en los programas de las pedagogías activas, con los de escuela y vida de las teorías curricularistas. Para los primeros, la acción educativa es un proceso de enriquecimiento de la experiencia que debe permitir el libre desenvolvimiento, en un orden psicológico, a partir de los intereses y la creación de las condiciones favorables a ellos por parte de la escuela. Para los segundos, se trata de una educación que prepara para la vida, en un orden lógico, prepara para actividades específicas.

Este diseño se entiende como la racionalización del currículo: se modifican las concepciones de aprendizaje, enseñanza y de la función del profesor. El aprendizaje, entendido como adquisición de habilidades, destrezas y comportamientos tendrá que ser evaluado en función de la medición del grado de cumplimiento de los indicadores establecidos; así, el profesor es el administrador del currículo. Por su parte, Zuleta (1995) sostiene que la concepción de currículo se ha reducido al plan de estudios y, como resultado, la autoridad política anula la libertad de pensamiento y "consolida una máquina burocrática que se dice pedagógica" (Zuleta, 1995, p. 138). No obstante, queremos aquí reconocer el mandato legal relacionado con la 'autonomía', pues es este el marco de acción para las instituciones a las que refiere el modelo educativo propuesto por la misión de sabios conformada a mitad de los años 1990: flexibles, contextualizadas y capaces de aprendizaje, donde los profesores deberán asumir posturas críticas y vigilantes.

La formación de docentes en pedagogías críticas (Díaz, 2008; Mejía, 2008) plantea poner en el primer plano la dimensión humana de la educación, en tanto práctica social capaz de ser objetivada por el pensamiento de un profesional de la educación con una sólida formación profesional y ética en su actuación, cuyo pensamiento esté orientado a garantizar el libre desarrollo de la personalidad y de los proyectos de vida de sus estudiantes y de las comunidades a las que ellos pertenecen. Un profesor soportado por una institucionalidad anclada en los proyectos de las comunidades y de los entornos para los que presta su función educadora, al tiempo que interpreta en clave de la construcción de redes simbólicas de conocimiento los desarrollos de las ciencias, las artes y las técnicas expresados en las propuestas curriculares que hace el país en pro del desarrollo individual y social.

El Estado colombiano ha buscado solucionar problemas como la desarticulación entre los modelos educativos y las problemáticas de los grupos étnicos: baja cobertura, ineficiencia, falta de pertinencia y dispersión geográfica, entre otros, mediante el establecimiento de la etnoeducación, que a partir de la Constitución Política de 1991 se ha configurado como una política pública, reglamentada por varias leyes (21 de 1991, 47 y 70 de 1993, 115 de 1994, 33 de 1997, entre otras) y decretos (804 y 2249 de 1995, 1122 de 1998, entre otros) que, aparte de sus disposiciones específicas, en general, declaran al Estado como garante del derecho a la educación de los grupos étnicos y estipulan que dicha educación debe estar acorde a sus "aspiraciones y necesidades etnoculturales" (Ley 70 de 1993).

La declaración afirmativa en la política pública ha hecho visibles en la configuración poblacional de la nación a los indígenas, gitanos, raizales y afrocolombianos, lo que constituye un reconocimiento formal en relación con sus derechos y la diversidad cultural del país. Como lo evidencian Rojas (1999) y Rojas y Castillo (2005), solo a partir de esta declaración y de la configuración de organizaciones de orden político y social, por parte de estos pueblos, ha sido posible advertir alguna participación suya en los estamentos estatales; entretanto, uno de los principales campos de lucha por la reivindicación de derechos ha sido el de la educación, en todos los niveles del sistema educativo. 
En tanto política, la etnoeducación se concreta como carrera profesional desde hace unos veinte años. Los estudiantes provenientes de grupos étnicos que acceden a la educación superior para formarse como maestros reciben el título de etnoeducadores o licenciados en etnoeducación, lo que les permite participar de convocatorias públicas para territorios especiales como resguardos indígenas y consejos comunitarios (poblaciones afrocolombianas) (Rojas y Castillo, 2005).

De manera tal que, por lo menos formalmente, en Colombia, las políticas educativas buscan responder nominalmente a las características, necesidades y aspiraciones de los grupos étnicos, desarrollando la identidad cultural, la interculturalidad y el multilingüismo. De acuerdo con el Ministerio de Educación Nacional (2001), la política de etnoeducación busca atender las siguientes problemáticas:

, La poca correspondencia del servicio educativo con la realidad de los pueblos;

, La inadecuada gestión administrativa;

> La deficiente infraestructura y dotación;

> La no pertinencia en formación y capacitación de docentes;

, La baja cobertura e ineficiencia;

, La poca coordinación intra e interinstitucional;

> La dispersión geográfica de la población objeto.

En la Ley 115, artículo 55, se define la etnoeducación como aquella:

Que se ofrece a grupos o comunidades que integran la nacionalidad y que poseen una cultura, una lengua, unas tradiciones y unos fueros propios autóctonos. Esta educación debe estar ligada al ambiente, al proceso productivo, al proceso social y cultural, con el debido respeto de sus creencias y tradiciones.

Establece como su finalidad "afianzar los procesos de identidad, conocimiento, socialización, protección y uso adecuado de la naturaleza, sistemas y prácticas de organización, uso de las lenguas vernáculas, formación docente e investigación en todos los ámbitos de la cultura" (Ley 115, artículo 56). Por otro lado, con la Ley 70 de 1993 (artículo 3) se legisló para las poblaciones afrodescendientes, teniendo como fundamento los siguientes principios:

El reconocimiento y la protección de la diversidad étnica y cultural y el derecho a la igualdad de todas las culturas que conforman la nacionalidad colombiana; el respeto a la integralidad y dignidad de la vida cultural de las comunidades negras; la protección del medio ambiente atendiendo a las relaciones establecidas por las comunidades negras con la naturaleza.

En términos generales, el enfoque de los programas de etnoeducación apunta a la construcción de una educación hacia la autodeterminación y reconocimiento de los pueblos, sus valores, normas, principios, espiritualidades, lenguas y costumbres, que orientan las visiones de vida y de mundo y se concretan en sus cosmovisiones, planes de vida (para el caso de comunidades originarias) y proyectos de etnodesarrollo (para el caso de comunidades afrodescendientes agrupadas en consejos comunitarios), los cuales equivalen a los planes de desarrollo. Por su parte, los mestizos campesinos carecen de este reconocimiento político, aunque se ven también beneficiados de esta modalidad educativa por su componente de desarrollo rural.

\section{Etnoeducación en la Universidad Pontificia Bolivariana}

Desde la Escuela de Educación y Pedagogía, la Universidad Pontificia Bolivariana forma etnoeducadores con una propuesta académica y pedagógica cuyas particularidades son representativas de la diversidad poblacional que atiende. En la actualidad, cuenta con estudiantes pertenecientes a 48 grupos étnicos, provenientes de 26 departamentos del país, entre indígenas, afrodescendientes y campesinos mestizos (Universidad Pontificia Bolivariana, 2019a). La licenciatura se ofrece en tres centros tutoriales situados en Puerto Asís, Putumayo; Toribío, Cauca y Medellín, Antioquia. 
Para la Universidad Pontificia Bolivariana (2019) la etnoeducación es un campo de saber interdisciplinario e intercultural, razón por la cual la propuesta pedagógica y curricular de la Licenciatura está relacionada con los conceptos de etnodesarrollo y bienestar de las comunidades. En palabas de Marín,

La relación entre etnodesarrollo y el concepto de bienestar se debe concretar en el respeto a un conjunto de derechos legítimos de los pueblos étnicos, codificados en el derecho internacional: Derechos políticos que permitan escuchar su voz y sus demandas, reconocer sus normas, acuerdos y autoridades propias; derechos jurídicos que enriquezcan el derecho positivo y las garantías individuales probados ancestralmente a través de normas y cargos; derechos sociales que posibiliten la libertad en la forma de organizarse, de elegir sus autoridades y de los medios para alcanzar una vida digna; derechos económicos que den pie al desarrollo autónomo de sus propios esquemas y alternativas de producción, organización para el trabajo y medios de comercialización; derechos culturales que estimulen su diversidad permitan la generación y multiplicación de usos y costumbres tradicionales, conocimiento y divulgación de sus cosmogonías, de sus medios de relación con la naturaleza y con la sociedad (Universidad Pontificia Bolivariana, 2019b).

La Universidad Pontificia Bolivariana, a partir de la declaración de su Modelo Pedagógico Integrado (2015), formula una propuesta de formación profesional de Licenciatura en Etnoeducación, circunscrita en el Proyecto Educativo Institucional (PEI) al principio curricular de la interculturalidad y como una acción de la función sustantiva de su proyección social. Curricularmente, la etnoeducación se propone como un campo de saber interdisciplinario e intercultural en el cual las relaciones de saberes de las ciencias se imbrican con los aspectos sociales y culturales de quienes hacen parte del proyecto formativo. De acuerdo con el Informe de Autoevaluación de la Licenciatura en Etnoeducación (Universidad Pontificia Bolivariana, 2019a), la interdisciplinariedad en el plan de estudios se concreta en la articulación de objetos y métodos de estudio de saberes: antropológicos, lingüísticos, de geografía e historia articulados por saberes de la investigación y la pedagogía.

En el mismo informe se concluye que el programa de etnoeducación de la UPB ejemplifica cómo una propuesta curricular, en el marco de un $\mathrm{PEI}$, hace posible poner en relación un sistema educativo con visiones de mundo, formas de vida, estilos de pensamiento, formas de crear conocimiento y aspiraciones de desarrollo social y humano de grupos sociales determinados. Es decir, como una oportunidad efectiva de educación para grupos étnicos en condiciones de vulnerabilidad que contribuye a la construcción de una cultura de paz.

\section{Recursos culturales como categoría teórica y metodológica}

El término 'recursos culturales' se acuña principalmente desde dos horizontes teóricos de la comunicación: los estudios culturales y la media ecology, los que, a su vez, generaron el paradigma interpretativo cultural de la comunicación, el cual posibilita entender que "el proceso comunicativo es base para la conformación de la cultura, en otras palabras, que la comunicación constituye el entorno cultural del ser humano y los usos y las prácticas culturales se revelan como experiencias de significación" (UPB y Corporación Región, 2016, p. 14). No obstante, la revisión de la literatura del campo teórico de la comunicación y de las ciencias sociales en general revela poco uso del término.

Por el contrario, la expresión 'recursos culturales' es recurrente en campos de conocimiento como el arte, la sociología de la cultura y la antropología, tanto su uso como su desarrollo conceptual se observan en mayor medida en los campos del turismo y el patrimonio. De hecho, en estos campos pueden encontrarse diversas clasificaciones de los recursos culturales (Vera y Dávila, 1995; Ito, 2003; Vásquez, 2004; Montero y Oreja, 2005; Mastromey y Wilches, 2007), destacando en estas la inclusión de bienes tangibles (museos, monumentos, edificios, calles, entre otros) e intangibles (danzas, costumbres, técnicas artesanales, etcétera). 
Las alusiones a bienes culturales, elementos culturales, productos culturales, actividades culturales, espacio cultural, equipamiento cultural, abundantes en los campos mencionados, pueden asociarse a una comprensión de los recursos culturales ligada al patrimonio cultural de comunidades, pueblos o naciones, entendido este como un repertorio de elementos tangibles e intangibles que hacen parte de la definición de la identidad de los grupos poblacionales y de su memoria colectiva. Por su parte, en 1979, la Unesco -que regula el patrimonio cultural de la humanidad- definía recursos culturales como:

El conjunto de bienes y objetos disponibles que permiten o facilitan la realización de actividades culturales, entendidas éstas en un sentido amplio que sobrepasa el concepto tradicional que las limita a aquéllas directamente relacionadas con las bellas artes. Bajo esta perspectiva, actividades culturales son aquéllas a través de las cuales los miembros de una sociedad se expresan, desarrollan sus potencialidades y expanden su personalidad (p. 1).

Y para el año 2017, Irina Bokova, entonces directora general de la Unesco, afirmaba que "debemos reforzar los recursos culturales de las ciudades, así como el patrimonio, el cual proporciona un sentido y una identidad a sus habitantes, y las oportunidades creativas capaces de fomentar la vitalidad, habitabilidad y prosperidad de las mismas" (Unesco, 2017, p. 5). De tal manera, resulta evidente no solo la vinculación directa del término y su desarrollo conceptual en los campos del patrimonio -específicamente, el patrimonio cultural y el turismo-, sino la valoración de los recursos culturales como una realidad presente y con incidencia en la vida de los grupos sociales.

Si se sigue la línea conceptual de los campos del patrimonio y del turismo, más los documentos de la Unesco, es posible hacer diversas clasificaciones de los recursos culturales que, a su vez, permitirían identificarlos de una manera muy concreta. Por ejemplo, recursos culturales materiales como lugares, monumentos, objetos, y recursos culturales inmateriales como costumbres, rituales, lenguas, por nombrar algunos. Ahora bien, en la cotidianidad de las personas se ponen en juego acciones, expresiones, gestos, valores y emociones, los cuales también constituyen recursos para entablar y mantener relaciones tendientes al buen vivir, al bienestar común, que superan las arbitrariedades de las relaciones de poder y las injusticias de la violencia.

Si bien en el propio término 'recursos culturales' puede atribuirse una valoración utilitarista de la cultura -crítica ampliamente desarrollada por George Yúdice ${ }^{4}$, en el proyecto Creando paz no se pretende la identificación de recursos culturales con mero ánimo de clasificación para una posterior explotación o gestión, sino persiguiendo la valoración, comprensión e interpretación de estos y el sentido que tienen para la construcción de tejido social y, por esta vía, para la construcción de cultura de paz, además del reconocimiento de su potencial formativo.

Es por ello que se adopta la definición sociosemiótica de la cultura que, en palabras de García Canclini (2004), "abarca el conjunto de los procesos sociales de significación, o, de un modo más complejo, (...) el conjunto de procesos sociales de producción, circulación y consumo de la significación en la vida social" (p. 34), definición que sigue la línea conceptual de Clifford Geertz (2005), quien propone la cultura como "sistemas en interacción de signos interpretables" y como un contexto dentro del cual pueden describirse de manera densa acontecimientos sociales, modos de conducta, instituciones o procesos sociales (p. 27). Como sostiene John Paul Lederach (2016):

Literalmente, las personas que viven en escenarios de violencia padecen y perciben la red de patrones y conexiones en la que están atrapadas. Perciben que personas, comunidades y redes, con sus actividades y acciones, están ligadas entre sí y contribuyen a formar patrones que pueden dar pie a acciones destructivas o constructivas (p. 84).

4 "El papel de la cultura se ha expandido de una manera sin precedentes al ámbito político y económico, al tiempo que las nociones convencionales de cultura han sido considerablemente vaciadas. En lugar de centrarse en el contenido de la cultura -esto es, el modelo de enaltecimiento (según Schiller o Arnold) o el de distinción o jerarquización de clases (según Bourdieu) que ofrecía en sus acepciones tradicionales, o sus más reciente antropologización como estilo de vida integral (Williams) conforme a la cual se reconoce que la cultura de cada uno tiene valor- tal vez sea más conveniente abordar el tema de la cultura en nuestra época, caracterizada por la rápida globalización, considerándola como un recurso" (Yúdice, 2002, p. 23). 
Haciendo eco a este mediador y pensador de la paz, "el cambio social exige atención cuidadosa a cómo se integra la gente, en su propio entorno, a espacios relacionales que proporcionan un lugar cálido, de entrada, algo apartado y, por lo tanto, seguro" (Lederach, 2016, p. 185). De tal manera, el parque, el sembrado, la cocina, el camino veredal, la olla comunitaria, constituyen espacios donde a través del encuentro cotidiano se trabaja la trama y la urdimbre del tejido social, lugares donde los lenguajes posibilitan estrechar lazos, plantear soluciones, construir sueños, compartir enseñanzas y aprendizajes. Estas experiencias significantes dan lugar a "espacios relacionales" que a su vez se configuran como recursos culturales. Identificar la "red de relaciones", como la nombra Lederach, que se construye en estos espacios, posibilita trascender el uso o vivencia cotidiana para encontrar en ella un potencial formativo sobre las maneras autóctonas de habitar, de convivir, de producir, de participar, de solucionar conflictos y de trazar rutas comunes.

Todo ello teniendo en cuenta que, como afirma Geertz (2005), el desarrollo biológico y el desarrollo cultural han estado imbricados, lo cual "conduce a la hipótesis de que los recursos culturales son elementos constitutivos, no accesorios, del pensamiento humano. (...) El sistema nervioso humano depende inevitablemente del acceso a estructuras simbólicas públicas para elaborar sus propios esquemas autónomos de actividad" (p. 82).

\section{Conclusiones}

El sistema educativo y los currículos se codeterminan, razón por la cual, tal como aboga la primera Misión de Sabios, se requiere del consenso entre los estándares de los sistemas occidentales, los regionales y los autóctonos (campesinos, afrodescendientes e indígenas). En este trabajo también se afirma que la construcción de cultura de paz pasa por las oportunidades efectivas que ofrece la educación para potenciar el desarrollo social sostenible y, en este sentido, que en el currículo de las pedagogías activas y críticas se entiende la acción educativa como proceso de enriquecimiento de la experiencia individual y colectiva, lo que demanda la creación de condiciones favorables para permitir el libre desenvolvimiento de la personalidad y de los proyectos colectivos (planes y proyectos de vida, proyectos de desarrollo comunitario o proyectos etnoeducativos).

La propuesta curricular del programa de formación de Licenciados en etnoeducación que ofrece la Universidad Pontificia Bolivariana en convenio con el Instituto Misionero de Antropología responde a demandas sociales y políticas que hacen grupos étnicos, en condiciones de ruralidad y marcada diferenciación social y cultural, al sistema educativo colombiano. Esta se plantea con una intencionalidad intercultural, es decir: valoración por lo diverso, reconocimiento de las capacidades y potencialidades de los grupos a los que está dirigida, involucra decisiones políticas con implicaciones en: las identidades -colectivas e individuales-, las formas de circulación y valoración de la comunicación y del conocimiento, las formas de vida, las lenguas que se hablan, los sentimientos que se expresan y de las distintas maneras de relacionamiento.

El estudiante de la Licenciatura en Etnoeducación tiene un bagaje vivencial que da cuenta de formas diferentes de enfrentar la vida -en comparación con el estudiante urbano- debido a las condiciones y características propios de sus entornos rurales, de selva, río o marítimos y de las vicisitudes propias de vivir en un país con muchas desigualdades; características que, a su vez, hacen de él una persona recursiva, práctica y con posibilidad de dar solución a problemas diversos y particulares.

Así, la definición de los perfiles de ingreso y de egreso del programa constituye una decisión de la gestión curricular crucial, al contemplar, más allá de habilidades de carácter académico tradicionales (lectura, escritura, inglés, manejo de tecnologías) el liderazgo social y comunitario como un requisito y una constante del proyecto educativo. Estos perfiles inciden sobre las concepciones y prácticas metodológicas que están fundamentadas en la contextualización, la aplicación del saber y su adecuación a situaciones reales y cambiantes; además, y quizás lo más relevante de enfatizar en el carácter colectivo de las identidades étnicas, enraizadas en las subjetividades expresadas en los proyectos de vida y comunitarios. 
La ecodignidad es el supuesto sobre el cual se sustenta la propuesta curricular para la etnoeducación, entendida como el reconocimiento de que es en la interacción naturaleza-ser humano-cultura como es posible la vida. El proyecto educativo se basa en la idea de que educarse en el respeto, el diálogo y el reconocimiento ${ }^{5}$ transforma la visión de mundo, desarrolla capacidades, incorpora valores ${ }^{6}$ que redundan en conductas que favorecen la manifestación de recursos culturales, es decir, del repertorio de elementos que hacen parte de la identidad y la memoria colectiva de los pueblos.

Por estas razones se sostiene aquí que la experiencia con la educación superior en programas como el de etnoeducación permite afirmar que los estudiantes, después de pasar por el proceso etnoeducativo, usan los recursos culturales para la construcción o reconstrucción del tejido social, y la formación profesional permite su representación o la metacognición, con lo cual se promueve el potencial formativo que les subyace para transformar formas de vida y aportar a los colectivos sociales a los que pertenecen.

Las palabras que se dicen, la forma en que se dicen; las maneras de aproximarse y acoger al otro; las maneras de relacionarse con la naturaleza y de aprovechar y respetar sus recursos; las expresiones sobre el territorio y las formas de recorrerlo y habitarlo; los proyectos productivos comunitarios; los lugares y las formas de reunión de las personas; los valores, proyectos, sueños y anhelos que se construyen y se transmiten a través de las conversaciones, constituyen signos interpretables presentes en la cotidianidad de las personas y grupos sociales.

Valorar todo aquello como recursos culturales contribuye a la idea de que "reelaborar el esquema de relaciones sociales es reacomodar las coordenadas del mundo experimentado" (Geertz, 2005, p. 38), especialmente, en el marco del proyecto Creando paz y de los estudiantes de Etnoeducación en Colombia, habitantes en su mayoría de lugares donde prevalece la violencia y quienes han sido afectados por el conflicto armado y por otro tipo de conflictos.

Precisamente, estos estudiantes y etnoeducadores demuestran con sus acciones, actitudes, expresiones y proyectos que cuentan con unos recursos, materiales e inmateriales, a los que al agregarles valor emocional dan origen a acciones constructivas con las cuales se contribuye al desarrollo de nuevas ciudadanías que se insertan en una cultura de paz.

\section{Referencias}

Albán, A. y Rendón, J. A. (2018). Crecimiento, desarrollo y democracia en Colombia. Bogotá: Ediciones de la $U$.

Ávila, R. (2019, 4 de diciembre). Desigualdad: un problema sin solución. El Tiempo. https://www.eltiempo.com/economia/sectores/desigualdad-como-esta-colombia-en-el-contexto-latinoamericano-439096

Colciencias. (2015). Convocatoria para proyectos de investigación en ciencias humanas, sociales y educación. Bogotá: Colciencias. https://bit.ly/2JRm007

Congreso de la República. (1993). Ley 70 de 27 de agosto por la cual se desarrolla el artículo transitorio 55 de la Constitución Política. Diario Oficial 41.013 de 31 de agosto de 1993. https://bit.ly/3kq22GW

Congreso de la República. (1994). Ley 115 de febrero 8 por la cual se expide la ley general de educación. Diario Oficial 41.214 de 8 de febrero de 1994. https://bit.ly/2K1tOlu

Díaz, E. (2008, 20 de agosto). La educación y los modos de subjetivación, dispositivos éticos y dispositivos disciplinarios. https://www.estherdiaz.com.ar/textos/educacion_subjetivacion.htm

\footnotetext{
5 Ver Taylor, Ch. (1993). El multiculturalismo y la política del reconocimiento. Ciudad de México: Fondo de Cultura Económica.

6 Ver Cortina, A. (2009). Ética de la razón cordial. Educar en la ciudadanía en el siglo XXI. Oviedo: Ediciones Nobel.
} 
García Canclini, N. (2004). Diferentes, desiguales y desconectados. Mapas de la interculturalidad. Barcelona: Gedisa.

Geertz, C. (2005). La interpretación de las culturas. Barcelona: Gedisa.

González, D. (2019, 22 de agosto). Educación para la movilidad social colombiana. La Libertad. https://diariolalibertad.com/sitio/2019/08/22/educacion-para-la-movilidad-social-colombiana/

Grupo de Memoria Histórica. (2013). ¡BASTA YA! Colombia: memorias de guerra y dignidad. Bogotá: Imprenta Nacional. https://bit.ly/2GUR6Dr

Instituto Colombiano para la Evaluación de la Educación (ICFES). (2015, agosto). Guía para la interpretación del Reporte de resultados en Saber Pro. Medidas de Aporte Relativo y otros indicadores de calidad de la Instituciones de Educación Superior.

http://www.icfesinteractivo.gov.co/aporteRelativolnstituciones/pdfs/guia.pdf

Ito, N. (2003). Intangible cultural heritage involved in tangible cultural heritage. En Place, memory, meaning: preserving intangible values in monuments and sites. 14th ICOMOS General Assembly and International Symposium. Victoria Falls, Zimbabwe.

https://www.icomos.org/victoriafalls2003/papers/A3-2\%20-\%20lto.pdf

Kauffman, C. (2015, febrero). ¿Existe la movilidad social en Colombia? Semana. Recuperado de https://www.semana.com/opinion/articulo/existe-la-movilidad-social-en-colombia-opinion-celeste-kauffman/416889-3

Lederach, J. (2016). La imaginación moral. El arte y el alma de la construcción de la paz. Bogotá: Semana Libros.

Linares, P. (2011). Reflexiones sobre la propuesta de reforma a la ley 30 de 1992: ¿fortalecimiento o debilitamiento de la autonomía universitaria? Pensamiento Jurídico, (31), 43-84.

https://revistas.unal.edu.co/index.php/peju/article/view/36725/39684

Luque, E., Gómez, V. M., Reyes, A., Ramírez, J., Carvajal, M. J. y Londoño, C. F. (1999). Hacia un sistema de oportunidades de formación para el trabajo. Una propuesta para Colombia. Bogotá: Edición preliminar. https://www.yumpu.com/es/document/read/13656145/hacia-un-sistema-de-oportunidades-de-formacion-

Martínez Boom, A. (1995). Tecnología educativa y modelo curricular en Colombia. En M. Villa, D. Restrepo y L. Escobar (Eds.), Educación y Sociedad (pp. 170-182). Medellín: Corporación Región, Corporación Penca de Sábila y Colegio Colombo Francés.

Mastromey, M. y Wilches, S. E. (2007). Los recursos culturales bajo la óptica contable. Comunicación presentada en XXVIII Jornadas Universitarias de Contabilidad. Universidad Nacional de Mar del Plata. Mar del Plata, Argentina, 7-9 noviembre de 2007. http://nulan.mdp.edu.ar/id/eprint/979

Mejía, R. (2008, mayo 22-24). Las pedagogías críticas en tiempos de capitalismo cognitivo. (Ponencia presentada en el encuentro Maestros Gestores, Pedagogías Críticas y Resistencias). Aletheia, 2(2). https://aletheia.cinde.org.co/index.php/ALETHEIA/article/view/27/24

Ministerio de Educación Nacional. (2001, abril). Etnoeducación una política para la diversidad. Altablero, 3. https://www.mineducacion.gov.co/1621/article-87223.html

Ministerio de Educación Nacional. (2007). Plan Decenal de Educación 2006-2016. Pacto social por la educación. Bogotá: Ministerio de Educación Nacional. https://bit.ly/2GYExqS 
Ministerio de Educación Nacional. (2017). Plan Nacional Decenal de Educación 2016-2026. El camino hacia la calidad y la equidad. Bogotá: Ministerio de Educación Nacional. https://bit.ly/38zCrsH

Montero, I. y Oreja, J. R. (2005). Diseño de un instrumento de medida para el análisis comparativo de los recursos culturales tangibles. Pasos, Revista de Turismo y Patrimonio Cultural, 3(2), 245-255. https://doi.org/10.25145/j.pasos.200503.019

Ospina, W. y Suárez, A. (s. f.). Reforma a la ley 30: Implicaciones a la educación superior pública colombiana (Ensayo académico en página web).

https://www.universidad.edu.co/reforma-a-la-ley-30-implicaciones-a-la-educacion-superior-publica-colombiana/

Rojas, T. (1999). La etnoeducación en Colombia: un trecho andado y un largo camino por recorrer. Colombia Internacional, 2.

https://pdfs.semanticscholar.org/917c/ef9e110e1f4b87549d090318720d5feba74e.pdf? ga $=2.48747814 .1670340604 .1617132054-602975555.1616467474$

Rojas, A. y Castillo, E. (2005). Educar a los otros. Estado, políticas educativas y diferencia cultural en Colombia. Cali: Editorial Universidad del Cauca.

Presidencia de la República y Colciencias. (1996). Informe de la Misión de los Sabios. Colombia: al filo de la oportunidad (Tomo I) (Versión PDF). Bogotá: Tercer Mundo. https://bit.ly/2UnC2Bv

Unesco. (1979). Modelo de encuesta sobre acceso a recursos culturales y realización de actividades culturales. París: Unesco. https://bit.ly/2Uj82Xd

Unesco. (2017). Cultura: futuro urbano. Informe mundial sobre cultura para el desarrollo urbano sostenible. París: Unesco. https://bit.ly/3njiESq

Universidad Pontificia Bolivariana. (2015). Modelo Pedagógico Integrado. https://www.upb.edu.co/es/documentos/doc-modelopedagogicoesn-lau-1464098892245.pdf

Universidad Pontificia Bolivariana y Corporación Región. (2016, 30 de marzo). Proyecto Creando Paz: recursos culturales en experiencias de mediación y gestión constructiva de conflictos y su aporte a la formación de competencias ciudadanas y construcción de cultura de paz. Sistema Integral de Gestión de Proyectos (Convocatoria 740-2015). Archivos del Departamento Administrativo de Ciencia, Tecnología e Innovación, Colciencias, Bogotá.

Universidad Pontificia Bolivariana. (2019a, noviembre). Informe de Autoevaluación. Medellín: Comité de Currículo de la Licenciatura en Etnoeducación.

Universidad Pontificia Bolivariana. (2019b, diciembre). Marco teórico. Aproximaciones y evidencias del impacto de los licenciados en etnoeducación en territorios de incidencia del programa (Documento de trabajo). Medellín: Jorge Hernán Marín.

Vásquez, R. (2004). Comercialización del turismo cultural. En $6^{\circ}$ Congreso de Turismo Universidad Empresa. Valencia, España.

Vera, J. F. y Dávila, J. M. (1995). Turismo y patrimonio histórico cultural. Estudios turísticos, (126), 161-177. http://www.academia.edu/download/43209483/citart.pdf

Yúdice, G. (2002). El recurso de la cultura. Usos de la cultura en la era global. Barcelona: Gedisa.

Zuleta, F. (1995). Currículo e ideologías: sus fronteras, escenarios y protagonistas. En M. Villa, D. Restrepo y L. Escobar (Eds.), Educación y Sociedad (pp. 136-144). Medellín: Corporación Región, Corporación Penca de Sábila y Colegio Colombo Francés. 